(C) 2005 International Press

Adv. Theor. Math. Phys. 9 (2005) 575-591

\title{
On naked singularities and the collapse of self-gravitating Higgs fields
}

\author{
Mihalis Dafermos \\ University of Cambridge, Department of Pure Mathematics and \\ Mathematical Statistics, Wilberforce Road, Cambridge CB3 0WB, \\ United Kingdom \\ M.Dafermos@dpmms.cam.ac.uk
}

\begin{abstract}
We consider the problem of collapse of a self-gravitating Higgs field, with potential bounded below by a (possibly negative) constant. The behaviour at infinity may be either asymptotically flat or asymptotically AdS. This problem has recently received much attention as a source for possible violations of weak cosmic censorship in string theory. In this paper, we prove under spherical symmetry that "first singularities" arising in the non-trapped region must necessarily emanate from the centre. In particular, this excludes the formation of a certain type of naked singularity which was recently conjectured to occur.
\end{abstract}

A fundamental open problem in classical general relativity is the problem of weak cosmic censorship, the conjecture that generic asymptotically flat initial data lead to a Cauchy development with a complete null infinity.

e-print archive: http://lanl.arXiv.org/abs/gr-qc 
In the case of a spherically symmetric scalar field with vanishing potential, the conjecture was resolved in the affirmative by Christodoulou [2]. Previously, Christodoulou had proven [6] that the caveat "generic" was necessary, by constructing explicit examples of naked singularities forming from the collapse of regular data. These naked singularities emanate from the centre.

It has been suggested recently $[15,17]$ that the situation changes drastically for self-gravitating Higgs fields with potentials which can take negative values. Such "non-classical" ${ }^{1}$ matter can be motivated by considerations arising from string theory. In $[15,17]$, the authors advance certain heuristic arguments to show that naked singularities not emanating from the centre can arise and in fact will arise for an open set of initial data.

In this paper, we will prove a simple estimate for self-gravitating Higgs fields with potential bounded below by a (possibly negative) constant. In the evolutionary context, this estimate shows that "first singularities" arising from the non-trapped region ${ }^{2}$ must necessarily emanate from the center. Since the past of infinity (in either the asymptotically flat or asymptotically AdS setting) must lie in the non-trapped region, ${ }^{3}$ this implies, in particular, that the naked singularities of [17] can, in fact, never arise.

Finally, in the case where the potential is in fact non-negative, then the results of this paper show that Higgs fields satisfy the assumptions of [11]. In particular, the existence of a single-trapped surface is sufficient to deduce the completeness of null infinity. The reader is referred to [11].

\section{Basic assumptions}

A self-gravitating scalar field with potential is described by a four-dimensional spacetime $(\mathcal{M}, g)$ and a function $\phi$ on $\mathcal{M}$ satisfying:

$$
\begin{gathered}
R_{\mu \nu}-\frac{1}{2} g_{\mu \nu} R=2 T_{\mu \nu}, \\
\phi_{; \mu}^{; \mu}=V^{\prime}(\phi), \\
T_{\mu \nu}=\phi_{; \mu} \phi_{; \nu}-\frac{1}{2} g_{\mu \nu} \phi_{; \alpha} \phi^{; \alpha}-g_{\mu \nu} V(\phi),
\end{gathered}
$$

where $V$ is a continuously differentiable function of its argument:

$$
V \in C^{1}(\mathbb{R}) .
$$

\footnotetext{
${ }^{1}$ Higgs fields with negative potential violate the dominant energy condition.

${ }^{2}$ This is the region of spacetime such that the outgoing expansion of the group orbit spheres is non-negative, whereas the ingoing expansion is negative.

${ }^{3}$ This fact only depends on the null convergence condition, which is still satisfied here.
} 
This paper will concern such spacetimes which are spherically symmetric, and evolutionary.

By spherically symmetric, we here mean that the group $S O(3)$ acts by isometry on $\mathcal{M}$ and preserves $\phi$. Actually, we will require something a little stronger, namely that

$$
\mathcal{Q}^{+}=\mathcal{M} / S O(3)
$$

inherits from $g$ the structure of a $1+1$-dimensional time-oriented Lorentzian manifold with boundary, with metric $\bar{g}$, such that

$$
g=\bar{g}+r^{2} d \sigma_{\mathbb{S}^{2}}
$$

where $d \sigma_{\mathbb{S}^{2}}$ here denotes the standard metric on $\mathbb{S}^{2}$ and $r$ is a non-negative function on $\mathcal{Q}^{+}$, the so-called area-radius. Since $\phi$ is constant on group orbits, it descends to a function defined on $\mathcal{Q}^{+}$.

By evolutionary, we mean that the spacetime is to have those properties one would require from the "unique future maximal development" of an appropriate initial or initial-boundary value problem for equations (1.1) to (1.3). We have two such problems in mind:

1. Initial data are defined on a complete asymptotically flat hypersurface, leading to an asymptotically flat maximal Cauchy development or alternatively

2. Initial data are prescribed on an asymptotically AdS hypersurface, supplemented with boundary conditions at infinity, leading to an asymptotically AdS spacetime.

Only in the former case, has the construction of such a maximal development been rigorously carried out. ${ }^{5}$ Nevertheless, the properties to be listed here must be satisfied for any reasonable notion of such an object.

We proceed here to list these properties. For the purposes of mathematical clarity, the reader can chose to consider the "properties" listed in this section as a priori assumptions.

\footnotetext{
${ }^{4}$ The study of spherical symmetric solutions of (1.1)-(1.3) with $V=0$ was initiated by Christodoulou [7]. See also [18] for general $V$.

${ }^{5}$ In particular, in this case, the assumption of spherical symmetry described above can be retrieved from an analogous assumption on initial data.
} 


\section{$1.1 \quad$ Geometry of $\mathcal{Q}^{+}$}

The boundary ${ }^{6}$ of $\mathcal{Q}^{+}$will consist of $\Gamma \cup S$, where $\Gamma$ is a connected timelike curve and $S$ is a connected spacelike curve, and $\Gamma \cap S$ is a single point, and such that $r(q)=0$ iff $q \in \Gamma$. We will call $\Gamma$ the centre. Let $L$ denote a null parallel vector field along $\Gamma \cup S$ pointing towards $\mathcal{Q}^{+} \cdot \mathcal{Q}^{+}$will be foliated by the family of null geodesics defined by $L$. We will call these geodesics "outgoing".

To discuss the behaviour of the boundary "at infinity", it is convenient to introduce what are essentially Penrose diagrams. Our quotient spacetime $\mathcal{Q}^{+}$will admit a $C^{3}$ map into a bounded subset of two-dimensional Minkowski space which preserves the causal structure. We henceforth identify $\mathcal{Q}^{+}$with its image under such a map. Standard null coordinates $u$ and $v$ provide a global coordinate chart on $\mathcal{Q}^{+}$, and the metric $\bar{g}$ can be written $-\Omega^{2} d u d v$. Let $u$ be such that constant $u$ curves are "outgoing", as defined above.

We assume that there exists a non-empty causal curve $\mathcal{I} \subset \overline{\mathcal{Q}^{+}} \backslash \mathcal{Q}^{+},{ }^{7}$ and that $\mathcal{Q}^{+}$is foliated by connected constant $v$ curves with past endpoint on $S$ or past end-limit point on $\mathcal{I}$. We call such null geodesics "ingoing".

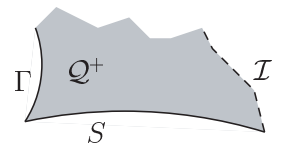

\subsection{Reduced equations}

From equations (1.1) to (1.3), we derive

$$
\begin{gathered}
\partial_{u} \partial_{v} r=-\frac{1}{r} \partial_{u} r \partial_{v} r-\frac{1}{4 r} \Omega^{2}+\frac{1}{2} r \Omega^{2} V(\phi), \\
\partial_{u} \partial_{v} \log \Omega=\frac{1}{4} \Omega^{2} r^{-2}+r^{-2} \partial_{u} r \partial_{v} r-\partial_{u} \phi \partial_{v} \phi, \\
\partial_{u} \partial_{v} \phi=-r^{-1} \partial_{u} \phi \partial_{v} r-r^{-1} \partial_{v} \phi \partial_{u} r-\frac{1}{4} \Omega^{2} V^{\prime}(\phi), \\
\partial_{u}\left(\Omega^{-2} \partial_{u} r\right)=-r \Omega^{-2}\left(\partial_{u} \phi\right)^{2}, \\
\partial_{v}\left(\Omega^{-2} \partial_{v} r\right)=-r \Omega^{-2}\left(\partial_{v} \phi\right)^{2} .
\end{gathered}
$$

\footnotetext{
${ }^{6}$ Recall that $\mathcal{Q}^{+}$is a manifold with boundary. The boundary is not quite a onedimensional manifold, but a piecewise regular curve.

${ }^{7}$ That is the set $\overline{\mathcal{Q}^{+}} \backslash \mathcal{Q}$ denotes the portion of the boundary of $\overline{\mathcal{Q}^{+}}$as as subset of $\mathbb{R}^{1+1}$, which is not part of its boundary, in the sense of manifolds-with-boundary.
} 
We assume here that $r, \Omega$, and $\phi$ are $C^{2}$ and that these equations hold pointwise.

\subsection{Local existence and extendibility criterion}

Evolutionary spacetimes are constructed by a local existence and uniqueness theorem in an appropriately defined function space. One first shows that there exists a non-empty "development" of initial data. Then, using the domain of dependence property, it is straightforward to show that there exists a unique maximal development.

Our embedding of $\mathcal{Q}^{+}$into $\mathbb{R}^{1+1}$ allows us to talk about its future boundary as a subset of $\mathbb{R}^{1+1}$. In what follows let $\overline{\mathcal{Q}^{+}}$denote the closure of $\mathcal{Q}^{+}$ in the topology of $\mathbb{R}^{1+1}$, and similarly $\overline{\mathcal{R}}$, etc., and let $J^{-}, J^{+}, D^{+}$, etc., refer to the causal structure of the $\mathbb{R}^{1+1}$. In this section, we shall formulate a criterion for a point $p \in \overline{\mathcal{Q}^{+}} \backslash(\overline{\mathcal{I}} \cup \bar{\Gamma})$ to be, in a suitable sense, a "first singularity". Since we are interested in considerations away from the centre, the following double null local existence result will be sufficient for our purposes.

Proposition 1.1. Let $\Omega, r$ and $\phi$ be functions defined on $X=[0, d] \times\{0\} \cup$ $\{0\} \times[0, d]$. Let $k \geq 0$, and assume $r>0$ is $C^{k+2}(u)$ on $[0, d] \times\{0\}$ and $C^{k+2}(v)$ on $\{0\} \times[0, d]$, assume that $\Omega$ and $\phi$ are $C^{k+1}(u)$ on $[0, d] \times\{0\}$ and $C^{k+1}(v)$ on $\{0\} \times[0, d]$, and assume that $V$ is a $C^{k+1}$ function of its argument. Suppose that equations (1.8), (1.9) hold initially on $[0, d] \times\{0\}$ and $\{0\} \times[0, d]$, respectively. Let $|\Omega|_{n, u}$ denote the $C^{n}(u)$ norm of $\Omega$ on $[0, d] \times\{0\},|\Omega|_{n, v}$ the $C^{n}(v)$ norm of $\Omega$ on $\{0\} \times[0, d]$, etc. Define

$$
N=\sup \left\{|\Omega|_{1, u},|\Omega|_{1, v},\left|\Omega^{-1}\right|_{0},|r|_{2, u},|r|_{2, v},|r|_{0}^{-1},|\phi|_{1, u},|\phi|_{1, v}\right\} .
$$

Then there exists a $\delta$, depending only on $N$, and a $C^{k+2}$ function (unique among $C^{2}$ functions) $r$ and $C^{k+1}$ functions (unique among $C^{1}$ functions) $\Omega$ and $\phi$, satisfying equations (1.5) to (1.9) in $\left[0, \delta^{*}\right] \times\left[0, \delta^{*}\right]$, where $\delta^{*}=$ $\min \{d, \delta\}$, such that the restriction of these functions to $[0, d] \times\{0\} \cup\{0\} \times$ $[0, d]$ is as prescribed.

The proof can be obtained by standard methods and is omitted. To describe the characterization of (part of) the boundary of $\mathcal{Q}^{+}$that this leads to, first let us introduce some terminology.

Definition 1.2. Let $p \in \overline{\mathcal{Q}^{+}}$. The indecomposable past subset $J^{-}(p) \cap$ $\mathcal{Q}^{+} \subset \mathcal{Q}^{+}$is said to be eventually compactly generated if there exists a compact subset $X \subset \mathcal{Q}^{+}$such that

$$
J^{-}(p) \subset D^{+}(X) \cup J^{-}(X) .
$$


Definition 1.3. A point $p \in \overline{\mathcal{Q}^{+}} \backslash \mathcal{Q}^{+}$is said to be a first singularity if $J^{-}(p) \cap \mathcal{Q}^{+}$is eventually compactly generated and if any eventually compactly generated indecomposable proper subset of $J^{-}(p) \cap \mathcal{Q}^{+}$is of the form $J^{-}(q)$ for a $q \in \mathcal{Q}^{+}$.

In particular, in view of the geometry of $\mathcal{Q}^{+}$, as described in Section 1.1, setting $p=\left(u_{s}, v_{s}\right)$, then if $p$ is a first singularity and if $p \notin \bar{\Gamma}$, then this implies that there exists an $\epsilon>0$ such that for any $u_{s}>u_{\epsilon}>u_{s}-\epsilon, v_{s}>$ $v_{\epsilon}>v_{s}-\epsilon$, the compact set

$$
X=\left\{u_{\epsilon}\right\} \times\left[v_{\epsilon}, v_{s}\right] \cup\left[u_{\epsilon}, u_{s}\right] \times\left\{v^{*}\right\}
$$

satisfies $X \subset \mathcal{Q}^{+} \backslash \Gamma$, and we have

$$
\left[u_{\epsilon}, u_{s}\right] \times\left[v_{\epsilon}, v_{s}\right]=D^{+}(X)=J^{-}(p) \cap D^{+}(X),
$$

and

$$
D^{+}(X) \cap \mathcal{Q}^{+}=D^{+}(X) \backslash\{p\} .
$$

Given a subset $Y \subset \mathcal{Q}^{+} \backslash \Gamma$, define

$$
N(Y)=\sup \left\{|\Omega|_{1},\left|\Omega^{-1}\right|_{0},|r|_{2},|r|_{0}^{-1},|\phi|_{1}\right\},
$$

where, for $f$ defined on $\mathcal{Q}^{+},|f|_{k}$ denotes the restriction of the $C^{k}$ norm to $Y$.

In the evolutionary context, Proposition 1.1 easily gives the following extension criterion.

Property 1.4. Let $p \in \overline{\mathcal{Q}^{+}} \backslash \bar{\Gamma}$ be a first singularity. Then for any compact $X \subset \mathcal{Q}^{+} \backslash \Gamma$ satisfying equation (1.10), we have

$$
N\left(D^{+}(X) \backslash\{p\}\right)=\infty .
$$

\section{$2 \mathcal{R}, \mathcal{T}$ and $\mathcal{A}$}

Let us introduce the notation:

$$
\begin{aligned}
& \nu=\partial_{u} r \\
& \lambda=\partial_{v} r .
\end{aligned}
$$

We define the regular region

$$
\mathcal{R}=\left\{q \in \mathcal{Q}^{+}: \lambda(q)>0, \nu(q)<0\right\},
$$

the trapped region

$$
\mathcal{T}=\left\{q \in \mathcal{Q}^{+}: \lambda(q)<0, \nu(q)<0\right\}
$$

and the marginally trapped region

$$
\mathcal{A}=\left\{q \in \mathcal{Q}^{+}: \lambda(q)=0, \nu(q)<0\right\}
$$


This notation derives from [4]. We call $\mathcal{R} \cup \mathcal{A}$ the non-trapped region.

In what follows, we will now always assume that the following hold:

Assumption 2.1. We have

$$
\partial_{u} r<0
$$

along $S$.

Property 2.1. For all $q \in \mathcal{I}$, for all $p \in J^{+}(q) \cap \mathcal{Q}^{+} \backslash I^{+}(q)$ and for all $R>0$, there exists a $p^{*} \in J^{+}(q) \cap J^{-}(p) \cap \mathcal{Q}^{+} \cap \backslash I^{+}(q)$ such that $r\left(p^{*}\right) \geq R$. Similarly, for all $q \in \mathcal{I}$, all $p \in J^{-}(q) \cap \mathcal{Q}^{+} \backslash I^{-}(q)$ and all $R>0$, there exists a $p^{*} \in J^{-}(q) \cap J^{+}(p) \cap \mathcal{Q}^{+} \cap \backslash I^{-}(q)$ such that $r\left(p^{*}\right) \geq R$.

Assumption 2.1 is motivated in [4]. Property 2.1 is perhaps the weakest possible notion that $\mathcal{I}$ is actually "at" infinity. Note that the set defined by $\mathcal{I}^{+}$in [11] for the asymptotically flat case clearly satisfies Property 2.1.

We have

Proposition 2.2. $\nu<0$ everywhere, i.e. $\mathcal{Q}^{+}=\mathcal{A} \cup \mathcal{R} \cup \mathcal{T}$.

Proof. The previous assumption, together with the global structure of $\mathcal{Q}^{+}$, as described in Section 1.1, implies that if $(u, v) \in \mathcal{Q}^{+}$, then there exists a $u^{*}<u$ such that $\nu\left(u^{*}, v\right)<0$. The proposition now follows immediately after integration of equation (1.8) along $\left[u^{*}, u\right] \times\{v\}$.

Similarly from equation (1.9), we immediately obtain,

Proposition 2.3. We have

$$
J^{-}(\mathcal{I}) \cap \mathcal{Q}^{+} \subset \mathcal{R}
$$

and

$$
\overline{J^{-}(\mathcal{I})} \cap \mathcal{Q}^{+} \subset \mathcal{R} \cup \mathcal{A}
$$

In particular,

Corollary 2.4. If $\mathcal{T} \neq \emptyset$, then $\mathcal{Q}^{+} \backslash J^{-}(\mathcal{I}) \neq \emptyset$. 


\section{The main theorem}

Let us introduce one final assumption:

Assumption 3.1. There exists a $C$ such that:

$$
V(x) \geq-C \text {. }
$$

The main theorem of this paper is

Theorem 3.1. Let $p \in \overline{\mathcal{Q}^{+}} \backslash \mathcal{Q}^{+}$be a first singularity. Then either

$$
p \in \bar{\Gamma} \backslash \Gamma
$$

or

$$
J^{-}(p) \cap \mathcal{Q}^{+} \cap D^{+}(X) \cap \mathcal{T} \neq \emptyset,
$$

for all compact $X$ satisfying equation (1.10).

\section{Proof of the main theorem}

It is equivalent to prove the following: Let $p \in \overline{\mathcal{Q}^{+}} \backslash \bar{\Gamma}$ be such that $J^{-}(p) \cap$ $\mathcal{Q}^{+}$is eventually compactly generated, and such that any compactly generated indecomposable subset $J^{-}(q) \cap \mathcal{Q}^{+} \subset J^{-}(p) \cap \mathcal{Q}^{+}$satisfies $q \in \mathcal{Q}^{+}$. Then $p \in \mathcal{R} \cup \mathcal{A}$.

Choose $\epsilon, X, u_{\epsilon}, v_{\epsilon}$, as in the statement following the definition of a first singularity, so that in addition $D^{+}(X) \backslash\{p\} \subset \mathcal{R} \cup \mathcal{A}$.

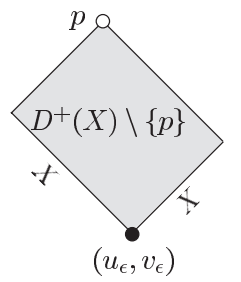

Let us introduce the notation:

$$
\begin{aligned}
& \zeta=r \partial_{u} \phi, \\
& \theta=r \partial_{v} \phi,
\end{aligned}
$$

and the quantity (in view of Proposition 2.2)

$$
\kappa=-\frac{1}{4} \Omega^{2} \nu^{-1} \text {. }
$$

We clearly have

$$
\kappa>0 .
$$


Key to our proof is the Hawking mass function, defined by

$$
m=\frac{r}{2}\left(1+4 \Omega^{-2} \partial_{u} r \partial_{v} r\right) .
$$

It is convenient to define also the so-called mass ratio

$$
\mu=\frac{2 m}{r} \text {. }
$$

By compactness of $X$, and the regularity assumptions of Section 1.2, it follows that $r, \kappa, \theta, \zeta, \phi, \lambda, \nu, m, \partial_{u} \Omega, \partial_{v} \Omega, \partial_{v} \lambda, \partial_{u} \nu$ are uniformly bounded above and below on $X$ :

$$
\begin{gathered}
0<r_{0} \leq r \leq R, \\
0 \leq \lambda \leq \Lambda, \\
0>\nu_{0} \geq \nu \geq-N, \\
|\phi| \leq P, \\
|\theta| \leq \Theta, \\
|\zeta| \leq Z, \\
|m| \leq M, \\
0<\kappa \leq K, \\
\left|\partial_{u} \Omega\right| \leq H, \\
\left|\partial_{v} \Omega\right| \leq H, \\
\left|\partial_{u} \nu\right| \leq H \\
\left|\partial_{v} \lambda\right| \leq H .
\end{gathered}
$$

In view of Property 1.4, it will be enough to show that uniform bounds similar to the above hold throughout $D^{+}(X) \backslash\{p\}$. 
The importance of the Hawking mass derives from the following identities (see [4]):

$$
\begin{gathered}
\partial_{u} m=\frac{1}{2}(1-\mu)\left(\frac{\zeta}{\nu}\right)^{2} \nu+r^{2} V(\phi) \nu \\
\partial_{v} m=\frac{1}{2} \kappa^{-1} \theta^{2}+r^{2} V(\phi) \lambda .
\end{gathered}
$$

To understand the nature of the terms in equations (4.10) and (4.11), note first that

$$
(1-\mu) \kappa=\lambda
$$

and thus

$$
1-\mu \geq 0
$$

on $\mathcal{R} \cup \mathcal{A}$. Equation (1.8) yields

$$
\partial_{u} \kappa=\frac{1}{r}\left(\frac{\zeta}{\nu}\right)^{2} \nu \kappa .
$$

From equation (4.13), and Proposition 2.2, it follows that the bound (4.5) holds throught $D^{+}(X) \backslash\{p\}$.

The idea now of the proof is as follows: As we shall see momentarily, the fact that $D^{+}(X) \backslash\{p\} \subset \mathcal{A} \cup \mathcal{R}$ immediately yields that the bounds (4.3) are preserved. If $V$ were non-negative, then the signs in equations (4.10) and (4.11) would immediately yield that (4.4) is preserved. In view of the bounds (4.3) on $r$ and (4.5) on $\kappa$, integration of equation (4.11) in $v$ would yield an $L^{2}(v)$ bound for $\partial_{v} \phi$. From this, bounds on all other quantities would follow in a straightforward manner.

In our case, $V$ is of course not non-negative. It turns out, however, that in view of Assumption 3.1, we can still derive estimates on $m$, since we can control the integral of the term with the "wrong" sign from equation (3.1) and the bounds (4.3) on $r .^{8}$ In view of the triangle inequality, we can still obtain an $L^{2}$ bound on $\partial_{v} \phi$ from our bounds (4.12) on $r$ and the bounds just obtained for $m$, upon integration of equation (4.11). We then continue as before.

\footnotetext{
${ }^{8}$ It is clear then that equation (3.1) is essential in this argument; indeed, if equation (3.1) is violated, there is no reason to believe that Theorem 3.1 holds.
} 
Now for the details: Given any point $\left(u^{*}, v^{*}\right) \in D^{+}(X) \backslash\{p\}$, the null curves $u=u^{*}$ and $v=v^{*}$ both intersect $X$ :

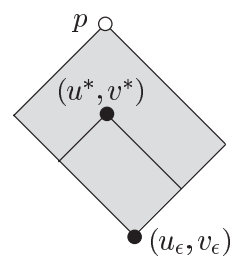

We proceed to obtain bounds for all quantities at $\left(u^{*}, v^{*}\right)$, independent of the choice of $\left(u^{*}, v^{*}\right)$.

Integrating equation (2.2) along $u=u^{*}$, in view of the inequality $\partial_{v} r=$ $\lambda \geq 0$ on $\mathcal{R} \cup \mathcal{A}$, we obtain

$$
r\left(u^{*}, v^{*}\right)=r\left(u^{*}, v_{\epsilon}\right)+\int_{v_{\epsilon}}^{v^{*}} \lambda\left(u^{*}, v\right) d v \geq r_{0},
$$

whereas integrating equation (2.1) along $v=v^{*}$, we obtain

$$
r\left(u^{*}, v^{*}\right) \leq R .
$$

Thus, the bounds (4.3) hold throughout $D^{+}(X) \backslash\{p\}$.

We now proceed to show a priori bounds for the mass:

$$
-\frac{R^{3} C}{3}-M \leq m \leq \frac{R^{3} C}{3}+M
$$

throughout $D^{+}(X) \backslash\{p\}$. Given $\left(u^{*}, v^{*}\right)$, we first show the right inequality of equation (4.14). Since $D^{+}(X) \backslash\{p\} \subset \mathcal{R} \cup \mathcal{A}$, we have - applying inequalities (3.1), (4.12) and $\nu<0$, to equation (4.10) - that

$$
\partial_{u} m \leq r^{2} V(\phi) \nu \leq-r^{2} C \nu
$$

in this region. Integrating the above inequality along $v=v^{*}$, yields

$$
m\left(u^{*}, v^{*}\right) \leq m\left(u_{\epsilon}, v^{*}\right)+\int_{u_{\epsilon}}^{u^{*}} \partial_{u} m \leq M+\frac{R^{3} C}{3}
$$

as desired. To show the left inequality of equation (4.14), we proceed similarly, i.e., from the inequality

$$
\partial_{v} m \geq r^{2} V(\phi) \lambda \geq-r^{2} C \lambda
$$

we obtain, integrating in $v$,

$$
m\left(u^{*}, v^{*}\right) \geq-\frac{R^{3} C}{3}-M .
$$

We have obtained, thus, that equation (4.14) indeed holds throughout $D^{+}(X) \backslash\{p\}$. 
From this bound on $m$, it now follows by integrating equation (4.11), and (4.10) that we have uniform a priori integral estimates:

$$
\begin{aligned}
& \left|\int_{u_{\epsilon}}^{u^{*}} \frac{1}{2}(1-\mu)\left(\frac{\zeta}{\nu}\right)^{2} \nu\left(u, v^{*}\right) d u+\int_{u_{\epsilon}}^{u^{*}} V(\phi) r^{2} \nu\left(u, v^{*}\right) d u\right| \\
& =\mid \int_{u_{\epsilon}}^{u^{*}} \frac{1}{2}(1-\mu)\left(\frac{\zeta}{\nu}\right)^{2} \nu d u+\int_{V(\phi) \geq 0} V(\phi) r^{2} \nu d u \\
& \quad+\int_{V(\phi)<0} V(\phi) r^{2} \nu d u \mid \leq \frac{2 R^{3} C}{3}+2 M
\end{aligned}
$$

and thus

$$
\begin{aligned}
\int_{u_{\epsilon}}^{u^{*}} & \frac{1}{2}(1-\mu)\left(\frac{\zeta}{\nu}\right)^{2}(-\nu) d u+\int_{V(\phi) \geq 0} V(\phi) r^{2}(-\nu) d u \\
& \leq \frac{2 R^{3} C}{3}+2 M+\frac{C R^{3}}{3}
\end{aligned}
$$

In view of the sign of $\nu$, and inequality (4.12), we have that both terms on the left are non-negative. We obtain in particular

$$
\int_{u_{\epsilon}}^{u^{*}} \frac{1}{2}(1-\mu)\left(\frac{\zeta}{\nu}\right)^{2}(-\nu)\left(u, v^{*}\right) d u \leq R^{3} C+2 M
$$

In an entirely similar fashion, we can obtain

$$
\int_{v_{\epsilon}}^{v^{*}} \frac{1}{2} \kappa^{-1} \theta^{2}\left(u^{*}, v\right) d v \leq R^{3} C+2 M .
$$

Integrating now equation (4.2), we obtain

$$
\begin{aligned}
\left|\phi\left(u^{*}, v^{*}\right)\right| & \leq\left|\phi\left(u^{*}, v_{\epsilon}\right)\right|+\left|\int_{v_{\epsilon}}^{v^{*}} \frac{\theta}{r}\left(u^{*}, v\right) d v\right| \\
& \leq P+\sqrt{\int_{v_{\epsilon}}^{v^{*}} \theta^{2} \kappa^{-1} d v} \sqrt{\int_{v_{\epsilon}}^{v^{*}} \frac{1}{r^{2}} \kappa d v} \\
& \leq P+2 \sqrt{R^{3} C+M} \sqrt{r_{0}^{-2} K \epsilon}=P_{b} .
\end{aligned}
$$

To estimate the first derivatives of $r$ and $\phi$, let us rewrite equations (1.5) and (1.7) as:

$$
\begin{gathered}
\partial_{v} \nu=\nu\left(2 \kappa\left(\frac{m}{r^{2}}-r V(\phi)\right)\right), \\
\partial_{u} \theta=-\frac{\zeta \lambda}{r}+\nu \kappa r V^{\prime}(\phi), \\
\partial_{v} \zeta=-\frac{\theta \nu}{r}+\nu \kappa r V^{\prime}(\phi),
\end{gathered}
$$


and let us denote by $C_{b}^{\prime}=\sup _{|x| \leq P_{b}} V^{\prime}(x)$ and $C_{b}=\sup _{|x| \leq P_{b}} V(x)$. These constants are finite in view of assumption (1.4).

Integrating equation (4.17), we obtain

$$
\begin{aligned}
\left|\theta\left(u^{*}, v^{*}\right)\right| \leq & \left|\theta\left(u_{\epsilon}, v^{*}\right)\right|+\left|\int_{u_{\epsilon}}^{u^{*}} \frac{\zeta \lambda}{r}\left(u, v^{*}\right) d u\right|+\left|\int_{u_{\epsilon}}^{u^{*}} r \nu \kappa V^{\prime}(\phi) d u\right| \\
\leq & \Theta+\left|\int_{u_{\epsilon}}^{u^{*}} \frac{\zeta}{\nu} \kappa \frac{(1-\mu) \nu}{r} d u\right|+R^{2} C_{b}^{\prime} K \\
\leq & \Theta+\sqrt{\int_{u_{\epsilon}}^{u^{*}}\left(\frac{\zeta}{\nu}\right)^{2}(-\nu)(1-\mu) d u \int_{u_{\epsilon}}^{u^{*}} \kappa^{2} \frac{(-\nu)(1-\mu)}{r^{2}}} d u \\
& +R^{2} C_{b}^{\prime} K \\
\leq & \Theta+2 K \sqrt{2 R^{3} C+2 M} \sqrt{r_{0}^{-1}+\left(\frac{R^{3} C}{3}+M\right) r_{0}^{-2}} \\
& +R^{2} C_{b}^{\prime} K=\Theta_{b} .
\end{aligned}
$$

Integrating equation (4.16) we obtain

$$
\begin{aligned}
\left|\log -\nu\left(u^{*}, v^{*}\right)\right| & \leq\left|\log \left(-\nu\left(u^{*}, v_{\epsilon}\right)\right)\right|+\left|\int_{v_{\epsilon}}^{v^{*}} 2 \kappa\left(\frac{m}{r^{2}}-r V(\phi)\right) d v\right| \\
& \leq \sup \left\{|\log N|,\left|\log \nu_{0}\right|\right\} \\
& +\left(2 K\left(r_{0}^{-2}\left(\frac{R^{3} C}{3}+M\right)+R C_{b}\right) \epsilon\right)=\log N_{b}
\end{aligned}
$$

while integrating equation (4.16) in $u$, since $\partial_{u} \lambda=\partial_{v} \nu$, we obtain

$$
\begin{aligned}
\lambda\left(u^{*}, v^{*}\right) & \leq \lambda\left(u_{\epsilon}, v^{*}\right)+2 K\left(r_{0}^{-1}\left(\frac{R^{3} C}{3}+M\right)+R^{2} C_{b}\right) \\
& \leq \Lambda+2 K\left(r_{0}^{-1}\left(\frac{R^{3} C}{3}+M\right)+R^{2} C_{b}\right) .
\end{aligned}
$$

Finally, integrating equation (4.18), we obtain that

$$
\begin{aligned}
\left|\zeta\left(u^{*}, v^{*}\right)\right| & \leq Z+\int_{v_{\epsilon}}^{v^{*}} \frac{|\theta \nu|}{r}\left(u^{*}, v\right) d v+\int_{v_{\epsilon}}^{v^{*}}\left|r \nu \kappa V^{\prime}(\phi)\left(u^{*}, v\right) d v\right| \\
& \leq Z+\Theta_{b} N_{b} r_{0}^{-1} \epsilon+R C_{b}^{\prime} \epsilon K N_{b} .
\end{aligned}
$$

We have estimated uniformly $\left|r^{-1}\right|,\left|\Omega^{-1}\right|,\left|\partial_{u} r\right|,\left|\partial_{v} r\right|,|\phi|,\left|\partial_{u} \phi\right|$ and $\left|\partial_{v} \phi\right|$ in $D^{+}(X) \backslash\{p\}$. By equation (4.16), we have clearly also estimated $\left|\partial_{u} \partial_{v} r\right|$. Thus, it remains only to estimate $\partial_{u} \partial_{u} r=\partial_{u} \nu, \partial_{v} \partial_{v} r=\partial_{v} \lambda, \partial_{u} \Omega, \partial_{v} \Omega$. 
These estimates, it turns out, are now quite straightforward. Differentiating equation (4.16) in $u$ we obtain,

$$
\begin{aligned}
\partial_{v}\left(\partial_{u} \nu\right)= & \partial_{u} \nu\left(2 \kappa\left(\frac{m}{r^{2}}-r V(\phi)\right)\right) \\
& +\nu\left(2 \partial_{u} \kappa\left(\frac{m}{r^{2}}-r V(\phi)\right)\right) \\
& +\nu\left(2 \kappa\left(\frac{\partial_{u} m}{r^{2}}-2 \frac{m}{r^{3}} \nu-\nu V(\phi)-V^{\prime}(\phi) \zeta\right)\right) .
\end{aligned}
$$

In view now of the bounds derived previously and (4.8), integrating this equation in $v$ immediately yields a uniform bound

$$
\left|\partial_{u} \nu\right| \leq \tilde{C}
$$

We leave to the reader explicit calculation of the constant. One argues similarly to obtain

$$
\left|\partial_{v} \lambda\right| \leq \tilde{C}
$$

On the other hand, integration of equation (1.6) in $u$ and $v$, respectively, in view of the bounds derived previously and the initial estimates (4.6) and (4.7), gives uniform bounds

$$
\left|\partial_{v} \Omega\right| \leq \tilde{C}
$$

and

$$
\left|\partial_{u} \Omega\right| \leq \tilde{C}
$$

Again, the details are left to the reader.

Thus, we have shown that

$$
N\left(D^{+}(X) \backslash\{p\}\right)<\infty .
$$

By Property 1.4, we have

$$
p \in \mathcal{Q}^{+} \text {. }
$$

By continuity of $r$ and $m$, it follows that

$$
p \in \mathcal{R} \cup \mathcal{A} \text {. }
$$

\section{Remarks on the global structure of spacetime}

From the above, one easily proves

Theorem 5.1. If $\mathcal{Q}^{+} \backslash J^{-}(\mathcal{I}) \neq \emptyset$, then there exists a null curve $\mathcal{H}^{+} \subset$ $\mathcal{R} \cup \mathcal{A}$, such that

$$
\mathcal{H}^{+}=\overline{J^{-}(\mathcal{I}) \cap \mathcal{Q}^{+}} \backslash\left(I^{-}(\mathcal{I}) \cup \overline{\mathcal{I}}\right)
$$


Proof. Consider the set $\mathcal{H}^{+}$defined by equation (5.1). Let $p \in \overline{\mathcal{Q}^{+}}$denote the future limit endpoint of $\mathcal{H}^{+} \cap \mathcal{Q}^{+}$. In view of Proposition 2.3, we clearly have

$$
\mathcal{H}^{+} \cap \mathcal{Q}^{+} \subset \mathcal{R} \cup \mathcal{A} \text {. }
$$

If $p \in \overline{\mathcal{I}}$, then there is nothing to show. If $p \notin \overline{\mathcal{I}}$ :

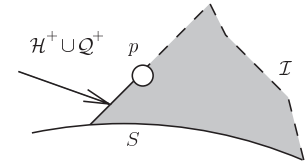

then it follows easily that $p$ is a first singularity. Clearly, $p \notin \bar{\Gamma}$, i.e. equation (3.2) does not hold. Since $J^{-}(p) \cap \mathcal{Q}^{+} \subset \overline{J^{-}(\mathcal{I})}$, it follows from Proposition 2.3 that $J^{-}(p) \cap \mathcal{Q}^{+} \subset \mathcal{R} \cup \mathcal{A}$. Thus, equation (3.3) does not hold either. We contradict the statement of Theorem 3.1.

Note that in view of Corollary 2.4, a sufficient condition for the assumption of Theorem 5.1 is $\mathcal{A} \cup \mathcal{T} \neq \emptyset$. Moreover, in view of Theorem 3.1, a sufficient condition for $\mathcal{T} \neq \emptyset$ is that there exists a first singularity $p$ such that $p \notin \bar{\Gamma}$, in particular, this is the case if "a component of the singularity is spacelike". Thus, it is clear that the naked singularities, as described in [15], can in fact never arise.

In general, we note that this argument says nothing about the behaviour of $r$ on the event horizon. In fact, a priori we could have $r \rightarrow \infty$, i.e., $\mathcal{H}$ could have a limit point on $\mathcal{I}$ itself. On the other hand, in the case of a Higgs field with non-negative potential ${ }^{9}$ collapsing from spherically symmetric asymptotically flat initial data, we can say more: Indeed, the results of this paper clearly imply that such fields satisfy the assumptions of [11]. It then follows from the results of [11] that if $\mathcal{Q}^{+} \backslash J^{-}(\mathcal{I}) \neq \emptyset$, then null infinity is complete (see below), and moreover a Penrose inequality holds bounding the area radius of the event horizon by twice the final Bondi mass, which is in turn finite.

\section{Weak cosmic censorship?}

Weak cosmic censorship is the conjecture that for generic initial data, $\mathcal{I}$ is complete. ${ }^{10}$ We have noted above that in the case $V \geq 0$ and $\mathcal{Q}^{+} \backslash$ $J^{-}\left(\mathcal{I}^{+}\right) \neq \emptyset$, the completeness of $\mathcal{I}^{+}$in the asymptotically flat setting

\footnotetext{
${ }^{9}$ In this case, the positive energy condition holds.

${ }^{10}$ See $[3,12]$ and compare with [14]. Completeness can be defined with respect to an appropriate induced connection on $\mathcal{I}$ related to the conformal compactifications given by the Penrose diagrams we are employing. For a definition of the notion of completeness applicable in the general (not necessarily spherically symmetric) asymptotically flat case,
} 
follows from [11]. See also [10] for decay results in the case $V=0$. We remark, here, that in the case where it is only assumed that $V \geq-C$, then a priori, infinity may be either complete or incomplete, despite the fact that $\mathcal{H}$ is regular. Understanding the global properties of these spacetimes, thus, requires further examination.

\section{Acknowledgment}

The author thanks Gary Horowitz, Mukund Rangamani and David Garfinkle for helpful discussions on a previous version of this paper. Since the original appearance of this paper on the gr-qc arxiv, the scenario of [15] has been updated, so as, in particular, not to be inconsistent with the results proven here. See [16] and references therein. See also [1, 13] for related numerical work. This research is supported in part by NSF grant DMS-0302748.

\section{References}

[1] M. Alcubierre, J.A. Gonzales, M. Salgado and D. Sudarsky, The cosmic censorship conjecture: Is it generically violated? gr-qc/0402045, 2004.

[2] D. Christodoulou, The instability of naked singularities in the gravitational collapse of a scalar field, Ann. Math. 149 (1999), no 1, 183-217.

[3] D. Christodoulou, On the global initial value problem and the issue of singularities, Classical Quant. Grav. 16 (1999), no. 12A, A23-A35.

[4] D. Christodoulou, Self-gravitating relativistic fluids: a two-phase model, Arch. Rational Mech. Anal. 130 (1995), no. 4, 343-400.

[5] D. Christodoulou, The formation of black holes and singularities in spherically symmetric gravitational collapse, Comm. Pure Appl. Math. 44 (1991), no. 3, 339-373.

[6] D. Christodoulou, Examples of naked singularity formation in the gravitational collapse of a scalar field, Ann. Math. 140 (1994), no. 3, 607653.

[7] D. Christodoulou, The problem of a self-gravitating scalar field, Comm. Math. Phys. 105 (1986), no. 3, 337-361.

[8] M. Dafermos, The interior of charged black holes and the problem of uniqueness in general relativity, Comm. Pure Appl. Math. 58 (2005), 445-504.

without a priori regularity assumptions at infinity, see [3]. For a comparison of the weak and strong cosmic censorship conjectures, see $[3,8,9]$. 
[9] M. Dafermos, Stability and Instability of the Cauchy horizon for the spherically-symmetric Einstein-Maxwell-scalar field equations, Ann. Math. 158 (2003), 875-928.

[10] M. Dafermos and I. Rodnianski, A proof of Price's law for the collapse of a self-gravitating scalar field, preprint, 2003, gr-qc/0309115. Invent. Math. 162 (2005), 381-457.

[11] M. Dafermos, Spherically symmetric spacetimes with a trapped surface, preprint, 2004, gr-qc/0403032. Class. Quantum Grav. 22 (2005), $2221-2232$.

[12] R. Geroch and G. Horowitz, Asymptotically simple does not imply assymptotically Minkowskian, Phys. Rev. Lett. 40, no 4, 1978 203-206.

[13] M. Gutperle and P. Kraus, Numerical study of cosmic censorship in string theory, hep-th/0402109, 2004. J. High Energy Phys. (2004), no. 4, pp. 014-024, (electronic).

[14] S.W. Hawking and G.F.R. Ellis, The large scale structure of space-time, Cambridge Monographs on Mathematical Physics, No. 1. Cambridge University Press, London-New York, 1973.

[15] T. Hertog, G.T. Horowitz, and K. Maeda, Generic cosmic censorship violation in anti de sitter space, Phys. Rev. Lett. 92 (2004) 131101.

[16] T. Hertog, G.T. Horowitz, and K. Maeda, Update on cosmic censorship violation in $A d S$, preprint 2004, gr-qc/0405050.

[17] G. Horowitz, Creating naked singularities and negative energy, hep-th/0312123, 2003.

[18] E. Malec, Self-gravitating nonlinear scalar fields, J. Math. Phys. 38 (7), 1993, 3650-3668. 
